\title{
THE SPHERICAL MODEL OF LOGARITHMIC POTENTIALS AS EXAMINED BY MONTE CARLO METHODS
}

\author{
CHJAN C. LIM \\ AND JOSEPH NEBUS
}

\begin{abstract}
Euler's equations for inviscid fluid flow are examined by a discretized version which represents the fluid in a Hamiltonian point-vortex problem on the surface of the unit sphere. The vorticities - the site strengths of these points are constrained to satisfy a spherical model, constraining the circulation to a hyperplane and the enstrophy to the surface of a hypersphere. The distribution of site strengths is allowed to vary according to a Monte Carlo Metropolis-Hastings algorithm.

With this model the dependence of the system - as measured by a tool called the mean nearest neighbor parity, by considering the energy of the system over a number of Monte Carlo sweeps, by considering the distance between the greatest and the least sites, and by the statistics of both the site values at a given number of sweeps and of a single site over a number of sweeps - on such parameters as the number of points, the statistical mechanics temperature, and the number of sweeps used in the simulation are examined.

It is consistently found that in negative statistical mechanics temperatures a solid-body rotational state is found. These negative temperature states correspond to the expected distribution of site values, to the mean nearest neighbor parity, and to the energy of the solid-body rotational state. The positive-temperature state is not as strongly organized a state, and some of the difficulties in distinguishing between chaotic and organized states in positive temperatures are outlined. Nevertheless it is established that the Monte Carlo algorithm is an effective and fast method to model this point-vortex system, and numerical evidence suggests that our expectation of finding a single phase transition, between positive and negative inverse temperatures, is supported experimentally.
\end{abstract}

\section{IntRoduction}

The problem of Euler's equations for the inviscid flow of an incompressible fluid on the surface of the sphere is a fascinating one, and one which can be rewritten through vortex methods to be a Hamiltonian problem. This allows the use of the rich set of tools designed to study dynamical systems to be applied. Using either a particle or spatial discretization procedure one can derive finite dimensional Hamiltonian problems for the inviscid evolution of vorticity in two dimension.

Onsager[10] proposed modeling the flow of a turbulent fluid through long-lived point vortices. The distribution of these point vortices would become a problem of finding a statistical equilibrium. This result depends upon the fluid modeled being inviscid, and on the ergodicity of the point vortices. If one writes the entropy of the system of point vortices as a function of energy then it is not a strictly increasing function. There is a critical energy past which the entropy begins to decrease; and this results in the phenomenon of negative temperatures. By Monte Carlo methods

\footnotetext{
${ }^{1}$ Contact address: limc@rpi.edu
} 
in which we approach a minimum of the inverse temperature times the system energy, this results at low energies - positive temperatures - in vortices of like signs repelling one another and those of opposite signs attracting. At the extremely high energies of negative temperatures the reverse happens, and vortices of like signs will cluster together. If the vortices are permitted to change their location this results in a clustering of like-signed sites. This negative temperature noted by Onsager is one of the theoretical basis of the so-called inverse energy cascade for two-dimensional turbulence. We expect that a similar observation will result from a lattice-based simulation of two-dimensional Euler statistics.

Since it is much easier to perform numerics on a compact surface such as the sphere, we will report on simulations of Euler statistics on the surface of the sphere. If one uses a spatial lattice decomposition of the vorticity field, the kinetic energy of fluid flow is again represented by a finite dimensional Hamiltonian function with a logarithmic kernel, but the robust conserved quantities such as total circulation and enstrophy become respectively the sum and sum of squares of the lattice site vorticity values. It was observed by the first author in [7] and [9] that the discrete form of the enstrophy is just the spherical constraint in a spin-lattice model known as the spherical model. The spherical model was introduced by Berlin and Kac as a model for ferromagnetism [1], and that is the purpose to which it has been most often applied. We will refer to this statistical mechanics model of $2 \mathrm{D}$ inviscid fluid flows as the energy-enstrophy-circulation theory since these are exactly the three conserved quantities.

In this paper a Monte Carlo method based on a suitable modification of the Markov Chain algorithm of Metropolis and Hastings [4] is employed to find several statistical equilibria of the spherical model or energy-enstrophy-circulation theory. Initially the Metropolis-Hastings algorithm is used to generate a mesh of points on the surface of the sphere which covers the domain in a reasonably uniform manner. Once this mesh is set, a new round of Monte Carlo is applied. Initially randomly chosen vorticities are given to each mesh site so that the spherical constraints are set. Then during this second round the vortices are given strengths which are allowed to vary, provided the circulation and enstrophy are held constant (the spherical model). From this a statistical equilibrium dependent on the inverse temperature $\beta$ is found.

A tool called the mean nearest neighbor parity is applied to determine in what state the statistical equilibrium is. The dependence of the mean nearest neighbor parity versus $\beta$ is then explored as the size of the mesh changes and as the system is allowed to evolve over time. These suggest the drawing of several conclusions about the behavior of the spherical model of logarithmic potentials, some of which may be checked against analytically known results.

We find that the numerical evidence points to the existence of a single phase transition between a parallel state for negative temperatures and an anti-parallel state at positive ones in the nonextensive thermodynamic limit [2]. The intermediate or chaotic state for a narrow range of $\beta$ on both sides of 0 , where the mean parity has generally small absolute values, can be interpreted as the unavoidable broadening of a phase transition in finite mesh simulations of critical phenomena [11]. Finally the parallel state found when the inverse temperature is negative is verified to be the solid-body rotational state as described by Lim [7]. 
In this paper we present a new Monte-Carlo Markov chain algorithm based on the Metropolis-Hastings method for updating the spins on a vortex lattice so as to simultaneously fix the values of two independent first integrals, namely the total circulation and enstrophy. We show that this algorithm is different from the common one of swapping vorticity values at a pair of sites, and can in principle reach all of the relevant phase space in the problem.

\section{MODEL}

The model under examination is derived from the Euler equation for inviscid fluid flow on the surface of the sphere. It examines the fluid flow by the familiar vortex representation of spatially decomposing the vorticity field into lattice site values. Thereafter site strengths or spins $s_{i}$ are allowed to vary subject to particular constraints. The fluid flow kinetic energy of the system is modulo a singular selfenergy term given by

$$
H(\vec{s}, \vec{x})=-\sum_{1 \leq i<j \leq N}^{N} s_{i} s_{j} J_{i, j}
$$

with $\vec{s}=\left(s_{1}, s_{2}, s_{3}, \ldots, s_{N}\right), \vec{x}=\left(\vec{x}_{1}, \vec{x}_{2}, \vec{x}_{3}, \ldots, \vec{x}_{N}\right)$ and $J_{i, j}$ the logarithm of the distance between $\vec{x}_{i}$ and $\vec{x}_{j}$ which on the surface of a sphere takes the form in (2).

Of particular interest in this vortex model are the discretized total circulation, $\sum_{i=1}^{N} s_{i}=\Gamma$, and enstrophy, $\sum_{i=1}^{N} s_{i}^{2}=\Omega$. Both quantities are discrete approximations to the first and second moments of the vorticity field in 2D Eulerian flows and so are conserved as the system evolves in real time [3]. Higher order moments of the vorticity are conserved in principle but not in the approximate finite dimensional models.

The Monte Carlo simulations done in this paper conserve both the discrete total circulation and the enstrophy and seek statistical equilibria of the lattice vortex system for a wide range of values of the inverse temperature $\beta$. This sets the numerical simulations to be a version of Kac's spherical model discussed in the previous section. Fixing the temperature of a statistical simulation is indicative of a method based on Gibbs canonical statistics, that is, the lattice system interacts with a virtual energy reservoir to reach equilibrium. However, because we also hold the total circulation and enstrophy fixed, these constraints are modeled microcanonically.

The Gibbsian statistics of the spherical model or the energy-enstrophy-total circulation theory are completely specified by sampling the system according to the probability measure

$$
P\left(\vec{s} ; \beta_{N}, \Omega, \Gamma\right)=\frac{1}{Z_{N}(\beta, \Omega, \Gamma)} \exp \left(-\beta_{N} H_{N}(\vec{s}, \vec{x})\right) \delta\left(\sum_{i=1}^{N} s_{i}^{2}-N\right) \delta\left(\sum_{i=1}^{N} s_{i}-\Gamma\right)
$$

where the sum in the partition function,

$$
Z_{N}\left(\beta_{N}, \Omega, \Gamma\right)=\sum_{\vec{s}} \exp \left(-\beta_{N} H_{N}(\vec{s}, \vec{x})\right) \delta\left(\sum_{i=1}^{N} s_{i}^{2}-N\right) \delta\left(\sum_{i=1}^{N} s_{i}-\Gamma\right)
$$

is taken over all lattice vortex vectors $\vec{s}$. Metropolis' algorithm for Monte Carlo simulation is based on a clever Markov chain method to generate this invariant measure [4]. The larger the size $N$ of the lattice, the more sharply peaked is the probability distribution around the most probable state $\vec{s}_{*}$ [11]. Thus, for fixed $\beta_{N}$ 
and fixed $N$ reasonably large, it will be a simple matter to run the algorithm and find the statistical equilibrium $\vec{s}_{*}$ with overwhelming probability $P\left(\vec{s} ; \beta_{N}, \Omega, \Gamma\right)$.

An effective simulation of a given Eulerian fluid flow on the sphere demands much more. For given enstrophy $\int_{D} \omega^{2}=\Omega>0$, we need to systematically increase the mesh size $N$ of the simulation to better approximate the continuum vorticity field $\omega$ that will give us the most probable state within the class of vorticity fields of zero total circulation and fixed enstrophy. While increasing the number of lattice sites from $N$ to $N^{\prime}$ we need to keep fixed the discrete enstrophy. Using a vorticity scale factor $\nu_{N}$ to describe the procedure, we have

$$
\nu_{N}^{2} \sum_{i=1}^{N} s_{i}^{2}=\nu_{N^{\prime}}^{2} \sum_{i=1}^{N^{\prime}} s_{i}^{2}=1
$$

by choosing

$$
\nu_{N}=N^{-1 / 2}
$$

and

$$
\sum_{i=1}^{N} s_{i}^{2}=N
$$

To see how to keep the kinetic energy constant as we let $N$ tend to $\infty$, we note that for a continuous vorticity field $\omega$,

$$
H=-\frac{1}{2} \int_{S^{2}} \int_{S^{2}} \omega(x) \omega(y) \log |1-x \cdot y| d A d A^{\prime} .
$$

Thus, for a uniform vorticity $\omega=\rho$,

$$
\begin{aligned}
H & =-\frac{\rho^{2}}{2} \int_{S^{2}} \int_{S^{2}} \log |1-x \cdot y| d A d A^{\prime} \\
& =-8 \pi^{2} \rho^{2}(\log 2-1) .
\end{aligned}
$$

Choosing this vorticity

$$
\rho=\frac{N \nu_{N}}{4 \pi}
$$

to be consistent with the enstrophy scaling, we get

$$
\begin{aligned}
H_{N} & =-8 \pi^{2} \frac{N^{2} \nu_{N}^{2}}{16 \pi^{2}}(\log 2-1) \\
& =-\frac{N}{2}(\log 2-1) \\
& \sim N .
\end{aligned}
$$

This motivates the temperature scaling below. We are obliged to use a properly scaled simulation inverse temperature $\beta_{N}=\beta / N$ in order to keep the simulated Gibbs factor $e^{-\beta_{N} H_{N}}$ constant when the lattice size $N$ is increased to model a vorticity field of fixed enstrophy. 
Collecting together the above scaling, we set [7]

$$
\begin{aligned}
\sum_{i=1}^{N} s_{i}^{2} & =N \\
\beta_{N} & =\frac{\beta}{N} \\
J_{i, j} & =\nu_{N}^{2} \log \left(1-\vec{x}_{i} \cdot \vec{x}_{j}\right) \\
\nu_{N} & =N^{-1 / 2}
\end{aligned}
$$

To verify more rigorously that these scalings do work, we will now calculate the energy directly. Since the simulated enstrophy is given by

$$
\Omega_{N}=\sum_{i=1}^{N} s_{i}^{2}=N
$$

this implies that the lattice site values $s_{i} \sim O(1)$. Then the energy

$$
H_{N}=-\frac{1}{2} \sum_{j}^{N} \sum_{k \neq j}^{N} J_{j k} s_{j} s_{k} \sim O\left(N^{2} \nu_{N}^{2}\right)=O(N)
$$

which agrees with the above rough estimate.

If we do a discrete Fourier transform based on the orthonormal set of eigenfunctions $Y_{m n}$ of the Laplace-Beltrami operator on the sphere $S^{2}$,

$$
z_{q}=N^{-1 / 2} \sum_{j=1}^{N} Y_{m n}^{*} s_{j},
$$

we find that the energy can be diagonalized,

$$
\begin{aligned}
H_{N} & =-\frac{1}{2} \sum_{j}^{N} \sum_{k \neq j}^{N} J_{j k} s_{j} s_{k} \\
& =-\frac{1}{N} \sum_{|q|=1}^{N} a(q)\left|z_{q}\right|^{2}
\end{aligned}
$$

where

$$
a(q)=O\left(\nu_{N}^{2}|q|^{-2}\right)
$$

are the eigenvalues of the energy interaction $J_{j k}$. It is well-known that the unscaled operator inverse to the Laplacian-Beltrami operator on the sphere has eigenvalues that behave like $O\left(|q|^{-2}\right)=O(N)$. By the fact that $z_{q} \sim O\left(N^{1 / 2}\right)$ (for $s_{j} \sim O(1)$ for all lattice size $N)$ and (4), we again find that $H_{N} \sim O(N)$. This Fourier diagonalization is essential for obtaining an exact expression of the partition function $Z$ of the spherical model in the limit as $N$ tends to $\infty$. In that calculation the term

$$
[p+\beta a(q)]^{-1}=\left[p+\frac{\beta}{N}|q|^{-2}\right]^{-1}
$$

arises and again we see that it is natural to introduce the scaled inverse temperature $\beta_{N}=\beta / N$.

This non-extensive scaling of the inverse temperature is also known to be correct for the thermodynamic limit of $2 \mathrm{D}$ vortex dynamics on a compact flow domain 
[2]. Between the compact domain, the minimum separation between sites, the requirement that total circulation is zero and enstrophy be fixed, there will exist a maximum possible energy achieved by this system. The existence of this maximum possible energy therefore justifies the exploration of negative temperatures.

\section{IMPLEMENTATION}

The first problem presented in implementing this problem is choosing what the mesh sites will be - what will be the fixed positions of the vortices. The goal is a mesh which is approximately uniform over the domain without being so regular as to make lattice artifacts likely. To achieve this, as well as to leave open the chance of using a different mesh on different experiments, a similar Monte Carlo simulation is run $[8]$.

Begin with a collection of $N$ points arbitrarily distributed on the sphere. Then run a Metropolis-Hastings simulation of how these points distribute themselves if each point represents a vortex of strength 1 . Working with a large positive inverse temperature of dispersal $\beta_{d}$, this seeks a minimum of the potential

$$
H(\vec{x})=-\sum_{1 \leq i<j \leq N}^{N} \log \left(1-\vec{x}_{i} \cdot \vec{x}_{j}\right)
$$

which is achieved by maximizing the product of the distances between pairs of particles. Each attempted move picks a site $i$ at random, chooses an axis of rotation $\vec{r}=\left(\sqrt{1-z_{i}^{2}} \cos (\theta), \sqrt{1-z_{i}^{2}} \sin (\theta), z_{i}\right)$ (with $z_{i}$ drawn uniformly from $[-1,1]$ and $\theta$ drawn uniformly from $[0,2 \pi)$ ), and an angle of rotation $\Delta \phi$ from $\left(0, \Delta \phi_{m}\right)$. The experiment accepts or rejects the rotation of point $\vec{x}_{i}$ by the angle $\Delta \phi$ around axis $\vec{r}$ based on the Metropolis rule.

After just a few hundred sweeps - one sweep being $N$ attempted moves of some site, and so representing a few hundred attempts to move each vortex - this will be a nearly but not quite symmetric pattern [8]. This is the mesh to use for this experiment. (The same mesh can be reused for multiple experiments, by setting the random number seed to the same number before generating the mesh, or by storing the mesh in an externally generated file and reading that at program startup. The advantage of reusing meshes is in the savings in startup time, and the greater ease with which measures such as the mean nearest neighbor parity discussed here may be compared; the disadvantage is any effects particular to one mesh will affect an entire run of experiments. That disadvantage may be overcome by repeating the experiments with new random number seeds for mesh generation, which is generally desired anyway.)

Many methods have been explored to generate meshes covering the sphere so that the distances between points are reasonably uniform, or so that the mesh has multiple symmetries, or can be generated from basic rules. These were not employed here partly out of concern that mesh effects might appear, but also because such deterministic methods very often are applicable only to select numbers of mesh points. An algorithm which can generate a very good covering of the sphere for 262 points may be completely unsuitable for 263 points. The Monte Carlo methods clearly have no such limitations. Another advantage they possess, though not employed in this paper, is that they may be applied to any compact domain, and with some modification can be applied to unbounded domains as well [8]. 
In the meshes generated for this paper the dispersal $\beta_{d}$ was taken to be $2,000,000$ and 4,096 complete sweeps were run before the mesh was used for further work. The maximum angle of rotation $\Delta \phi_{m}$ was 0.10 .

With this mesh of points $\vec{x}_{1}, \vec{x}_{2}, \ldots, \vec{x}_{N}$ then the spherical model simulation may begin. An initial configuration of site values $s_{i}$ are assigned. These are set to satisfy

$$
\begin{aligned}
& \sum_{i} s_{i}=\Gamma \\
& \sum_{i} s_{i}^{2}=\Omega
\end{aligned}
$$

for the designated initial circulation $\Gamma$ and initial enstrophy $\Omega$.

In this second stage of Monte Carlo simulation a sweep is $N$ attempted changes of site values. In order to preserve circulation and enstrophy at all times each attempted change must involve three sites. Given three sites $s_{1}, s_{2}$, and $s_{3}$ the attempted change is

$$
\begin{aligned}
s_{1}^{\prime} & =s_{1}-\delta-\epsilon \\
s_{2}^{\prime} & =s_{2}+\delta \\
s_{3}^{\prime} & =s_{3}+\epsilon
\end{aligned}
$$

with the requirements that

$$
\begin{aligned}
\delta & =\frac{(K+1) \cdot s_{1}-s_{2}-K \cdot s_{3}}{K^{2}+K+1} \\
\epsilon & =K \cdot \delta
\end{aligned}
$$

for a value of $K$ chosen at random from $[-1,1]$.

If $K$ is held equal to zero, these attempted changes involve just two sites; however, the proposed move is then equivalent to the swapping of the values of $s_{1}$ and $s_{2}$. Though valid this leaves the Metropolis-Hasting algorithm to explore only the permutations of the initial site values. Letting $K$ vary (and it is only programming convenience that keeps it from varying over all real numbers) allows the exploration of all configurations preserving circulation and enstrophy.

Only a few hundred sweeps are needed before the simulation settles into one of the states discussed in section 4 . For the simulations in this paper, besides a calibrating run of 1,000, 000 sweeps used to estimate how many sweeps are needed until the measured quantities settle to values near their long-term equilibrium values, 20, 000 sweeps were completed. This will be further explored experimentally in section 4 .

Measuring which state the system is in requires some tool. We expect to describe a state how likely it is any one site is surrounded by vortices of the same or of opposite signs. This is analogous to the spin-spin correlations which are useful in the Ising model, which need to be generalized to handle site values $s_{i}$ which are not necessarily plus or minus one, and to address a mesh which is not generally organized.

Call the parity of any two sites $i$ and $j$ be the product of the signs of $s_{i}$ and $s_{j}$. (If either site has vorticity zero their parity is zero.) The nearest neighbor parity for site $i$ is the parity of $i$ and whatever site (or, potentially, collection of sites) is nearest $i$. The mean nearest neighbor parity is the arithmetic mean of all these nearest neighbor parities. Despite its ease of implementation and the apparently clear match between the number and the intuitive sense of what state a system is in 
there are limitations which must be observed in applying this tool, some of which will be discussed later in this paper.

One more convenient tool is a histogram of either the site values $s_{i}$ after a certain number of sweeps, or of a single site's values over the course of the experiment. This cannot be used by itself to determine the state of a system, but historical data over many sweeps using this information can be informative, particularly when the mean nearest neighbor parity is also calculated.

Another measuring tool is to measure the distance from the 'north vorticity pole,' the site with greatest vorticity, to the 'south vorticity pole,' the site with least vorticity. As with the histogram data it is most informative when it can be viewed over the course of an experiment, rather than serving as a measure for a single collection of site values.

\section{Results}

Lim suggests analytically [6] [7] that we may expect up to three phases for this model, which can be observed numerically.

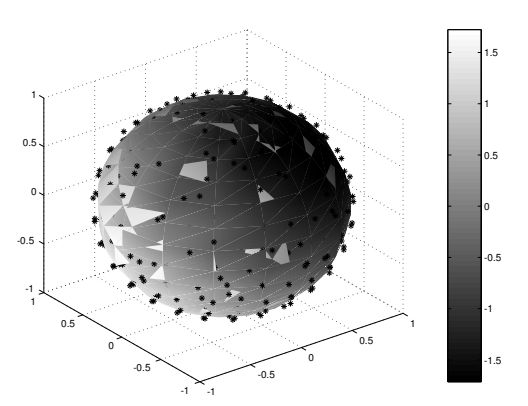

a. Parallel State.

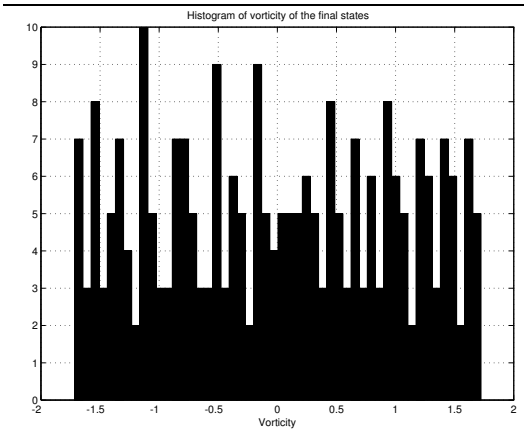

c. Final site histogram.

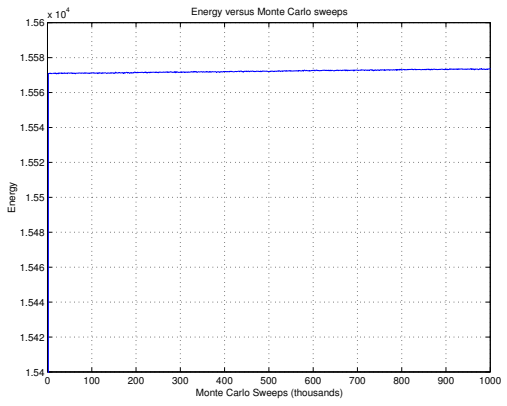

b. Energy versus sweep count.

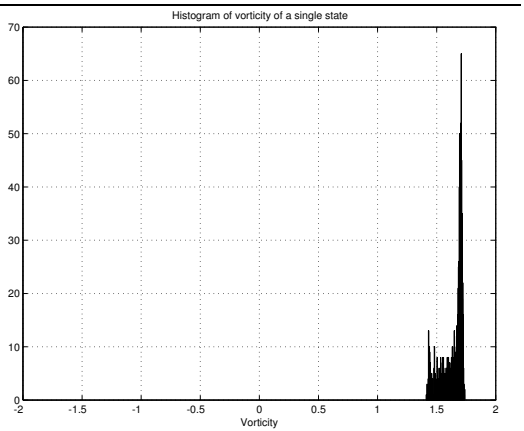

d. Single site histogram.

FiguRE 1. An example of the parallel state. For this example there are 256 points, with $\beta=-10$ and run for $1,000,000$ sweeps.

First is the parallel state (figure 1), which is only observed for negative inverse temperatures $\beta<0$. In this state any vortex is most often surrounded by vortices of the same sign. This is a high-energy state, and the mean nearest neighbor parity is near one. Examining a histogram of site values after a fixed number of sweeps (figure $1 \mathrm{c}$ ) shows the site values seem distributed approximately uniformly from 
a minimum to a maximum value. We have reason to believe this mode will be approximately that of the first spherical harmonic, a constant times the cosine of the angle between a site and the north vorticity pole. An individual site's value (figure $1 \mathrm{~d}$ ) is dominated by a sharp peak, indicating where the site's values have settled.

The distance between the north vorticity pole and the south vorticity pole in this state tends to be well-behaved (see figure 5). It is favorable for the energy to maximize the product $s_{i} s_{j}$ for neighboring sites, so that strong vortices of either sign are surrounded by other strong vortices of like sign. The result is that the distance between the greatest positive and the greatest negative sites approaches 2. That there remains some fluctuation in this distance as the number of sweeps continues is a reflection that neighboring sites tend to have similar magnitudes.

The plots of site vorticities over the sphere included in this section are of a Voronoi-type diagram: the shading of a point reflects the intensity of the mesh site nearest it. This appeared to be the simplest way in which to illustrate the vorticities.

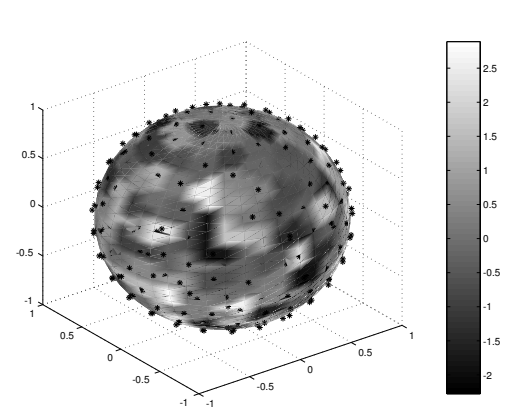

a. Chaotic State.

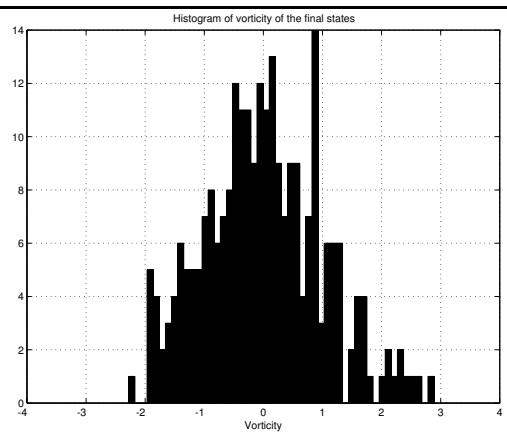

c. Final site histogram.

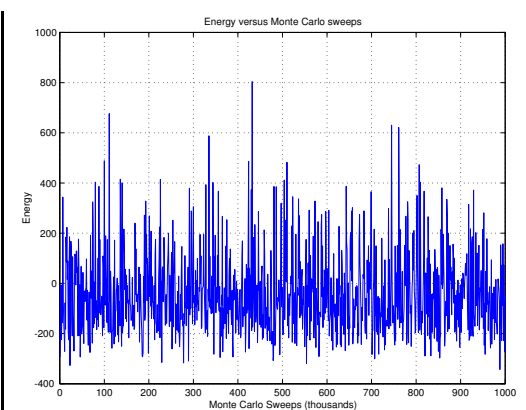

b. Energy versus sweep count.

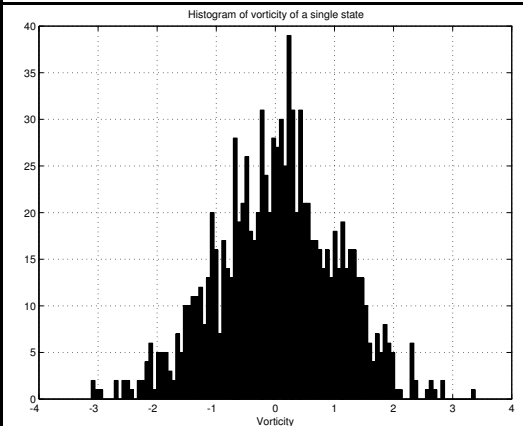

d. Single site histogram.

Figure 2. An example of the chaotic state. For this example there are 256 points, with $\beta=0$ and run for $1,000,000$ sweeps.

In the chaotic state - seen when the inverse temperature $\beta$ is near zero - there is no particular alignment among vortices (figure 2). The energy in this case never settles, and it fluctuates wildly while the simulation runs. Examining the site values after a fixed number of sweeps (figure $2 \mathrm{c}$ ) finds them tending towards a Gaussian 
distribution with a large standard deviation; examining the histogram of a single site's values over time (figure $2 \mathrm{~d}$ ) finds a similar range.

Here too the distance between the north and south vorticity poles may be measured (figure 5); it will tend to fluctuate wildly and never settle around any value. There is little reason for any one site to remain either vorticity pole, so that both wander over the surface of the sphere freely.

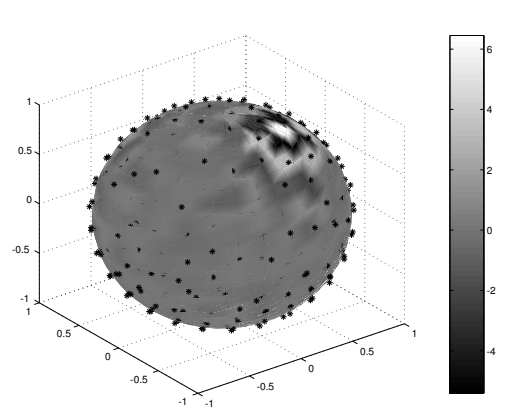

a. Antiparallel State.

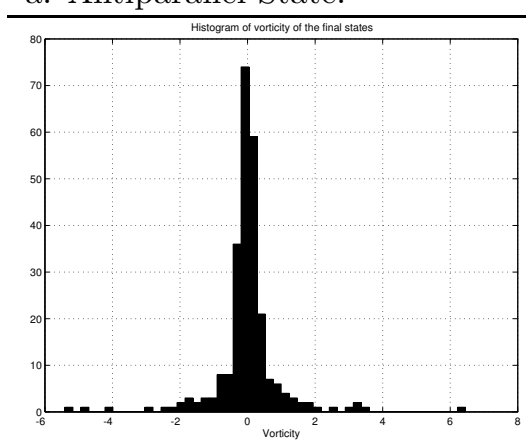

c. Final site histogram.

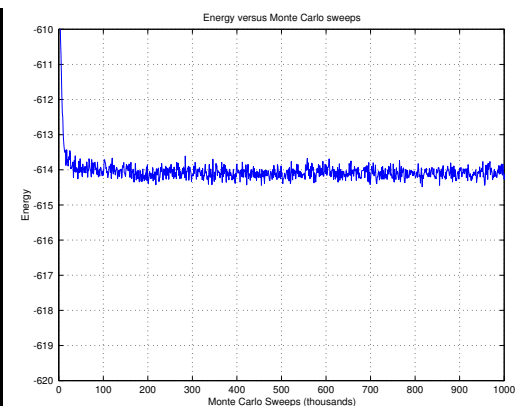

b. Energy versus sweep count.

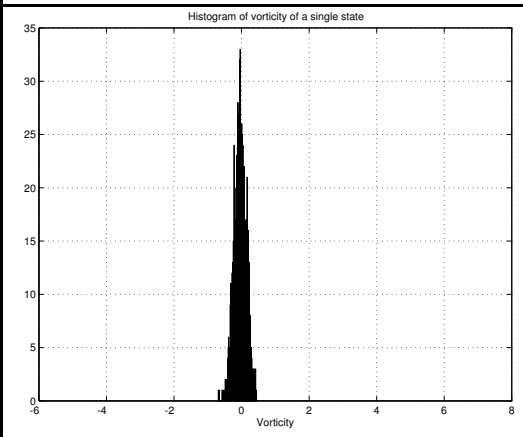

d. Single site histogram.

Figure 3. An example of the antiparallel state. For this example there are 256 points, with $\beta=10$ and run for $1,000,000$ sweeps.

The antiparallel state is a low-energy state, in which each vortex is surrounded by ones of the opposite vorticity (figure 3). While a histogram of all the site values after a fixed number of sweeps (figure $3 \mathrm{c}$ ) finds a Gaussian distribution with wide standard deviation, the examination of a single site's values (figure $3 \mathrm{~d}$ ) in time shows a smaller deviation.

The behavior of the distance between north and south vorticity poles is more interesting in this case than it is for the parallel or the chaotic states: it often shows a pattern of small fluctuations interrupted by large jumps (though they are obscured in the simulation used to generate figure 5). The process here is that each site will tend to have a nearest neighbor of opposite sign, and the Metropolis-Hastings algorithm's tendency to reduce the energy when $\beta$ is positive makes states in which $s_{i} s_{j}$ is minimized more probable. This pairwise product can be maximized, when the enstrophy has to stay constant, by having $s_{i}$ and $s_{j}$ of roughly equal magnitude and opposite sign. The result is the antiparallel state tends to create 'dipoles,' neighboring pairs of approximately equal magnitude and opposite sign. 
Generally, multiple dipole pairs will tend to be created, and they will tend to have approximately equal sizes. Often there will even be a chain of alternating signs and roughly equal magnitude over a good segment of the sphere. (The example plotted in figure 3 lacks a good long chain, but it does have a checkerboard-like pattern of positive and negative in the upper right of the picture, showing these dipoles collected into a quite organized island.) The result is the minor fluctuations in site values along these multiple dipole pairs will make either vorticity pole jump among several nearly equal sites. The result is a progression of times when the same two sites (not necessarily in a single dipole pair) are the vorticity poles, with sudden jumps when another site's magnitude grows enough to become one of them.

The expectation is that nearest neighbors will have opposite sign, and the drive to minimize energy while holding enstrophy constant encourages the nearest neighbors to have roughly equal magnitude. This indicates that we may expect - if we assume the north and south poles will eventually settle around one dipole of nearest neighbors - the distance to approximate $\frac{2 \pi}{N}$, roughly the distance of any neighboring pair of vortices. In the example used for figure 3 the minimum distance is 0.022 ; as $\frac{2 \pi}{256}$ is 0.025 this reinforces our supposition that the mesh generated is close to uniform and the north and south vorticity poles are being drawn as close together as possible.

(An interesting side effect of the spherical model is that the standard deviation of the site vorticities at any one sweep is a function of the enstrophy, circulation, and number of sites. It is independent of the Monte Carlo process and of what state the system is in.)

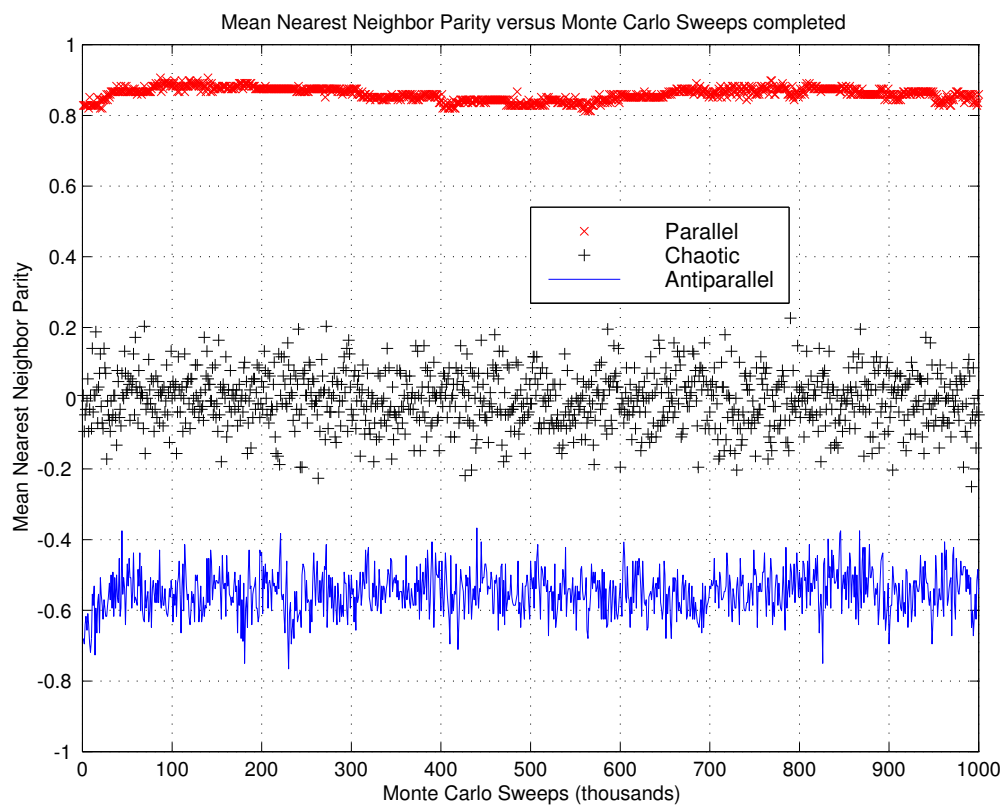

FiguRE 4. Mean nearest neighbor parity versus $\beta$ at a fixed mesh size $N$ over $1,000,000$ sweeps. 


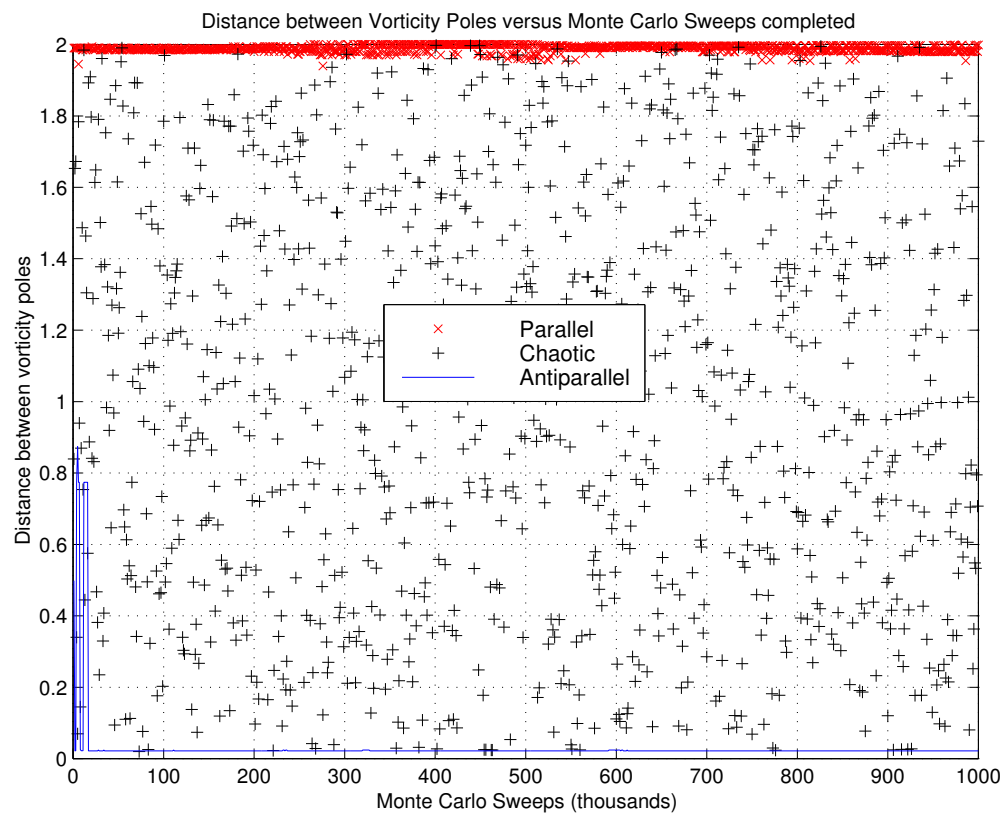

FiguRE 5. Distances between north and south vorticity poles versus sweep count for the parallel, chaotic, and antiparallel states for $1,000,000$ sweeps.

Considered now is the effect on the mean nearest neighbor parity of the number of sweeps completed. This is needed to establish in part how long the experimental runs need to be in order to have an estimate of the system's state measured correctly. Figure 4 presents the evolution of the mean nearest neighbor parity of a system with 256 points and circulation 0 run for 1,000,000 sweeps.

The first question is whether this change in the mean nearest neighbor parity as the sweep count increases reflects the system settling into its final state, and what that state ultimately is. For negative $\beta$ the mean nearest neighbor parity over time (excluding the first data point, which was the initial random assignment) has a mean of 0.86 with a standard deviation of 0.04 . (These numbers are rather consistent across multiple runs and different meshes.) The mean nearest neighbor parity for that mesh with site values assigned to match those of the first spherical harmonic (which we expect the system approaches) is 0.8359 .

(Another interesting point indicating the uniformity of the meshes generated by the Metropolis-Hastings algorithm here is that multiple meshes of 256 points were generated, each with different seed values. The 'spherical harmonic' mean nearest neighbor parity for each of them was close to 0.836 despite the meshes not being identical.)

In positive $\beta$ the mean nearest neighbor parity fluctuates more, and one may fairly question whether the value is settling at all. Here some statistical examination of the mean nearest neighbor parity evaluated at different times is useful: a histogram of mean nearest neighbor parity values at these different sweep counts reveals them to appear reasonably close to a bell curve. For this particular run 
at positive $\beta$ the mean of the mean nearest neighbor parities after the first data point is -0.55 with a standard deviation of 0.06. (As above the mean and standard deviation are rather consistent across multiple runs and different meshes.)

We try to explain this consistently greater fluctuation in the mean nearest neighbor parity versus sweeps completed for positive $\beta$ than for negative $\beta$. Doing so highlights one of the problems in relying solely on the mean nearest neighbor parity for studying the system.

Obviously the mean nearest neighbor parity measures only the sole neighbor closest to a site. Consider an antiparallel state which has three sites near the vertices of an equilateral (spherical) triangle; let two be positive and one negative. If the longer leg is only marginally longer than the other pairs there is only a weak preference to have a positive and negative pair on the shortest of the legs, with a parity of -1 , rather than to have both positives on that shortest leg, with a parity of 1 .

(There is also that the Monte Carlo method as programmed is unlikely at any one experiment to attempt a move which would invert the signs of the two "misaligned" sites. A sufficiently long run will eventually try swapping them, although the interactions with other neighboring sites may not guarantee that it will be approved.)

This effect does not occur in the parallel state where all points nearby any one site - except for the relatively few points on the 'equator' between positive and negative regions - are of the same sign. (Note that a greater fraction of points are near the equator when there are few mesh sites than when there are many; and as would be expected, the mean nearest neighbor parity is on average closer to zero for these smaller meshes and closer to 1 for larger ones.) Consequently the antiparallel state will have a greater number of sites which are 'incorrectly' aligned for the state.

One method which could improve this measure would be to identify all the neighbors of each site, and take the mean neighbor parity over all its adjacent sites. Nevertheless the mean nearest neighbor parity approximates its long-term mean value after as few as 20,000 sweeps. We may take its value, with the recognition that there must be an error margin whose size decreases as the number of mesh sites $N$ increases, to be approximately the equilibrated mean nearest neighbor parity.

The mean nearest neighbor parity is plotted as a function of inverse temperature $\beta$ for different mesh sizes in Figures 6 and 7 . The temperature scales are different for negative and for positive $\beta$, which reflects the different scales on which interesting behavior appears to occur.

There is a dependence of the parity on $N$ for negative $\beta$. As the number of points in the mesh increases the mean nearest neighbor parity increases, approaching 1 , and while the parity does eventually fall towards zero the inverse temperature at which it falls approaches zero. The suggestion is then that as the mesh size increases the parity approaches 1 for all negative temperatures - which is to be expected, as the parallel state is a high-energy state and this is characteristic of negative temperatures.

The dependence of the parity on $N$ for positive $\beta$ is almost identical. At $\beta=0$ the system is chaotically arranged; as $\beta$ increases the mean nearest neighbor parity drops towards about -0.5 . From the considerations mentioned above it is unlikely 


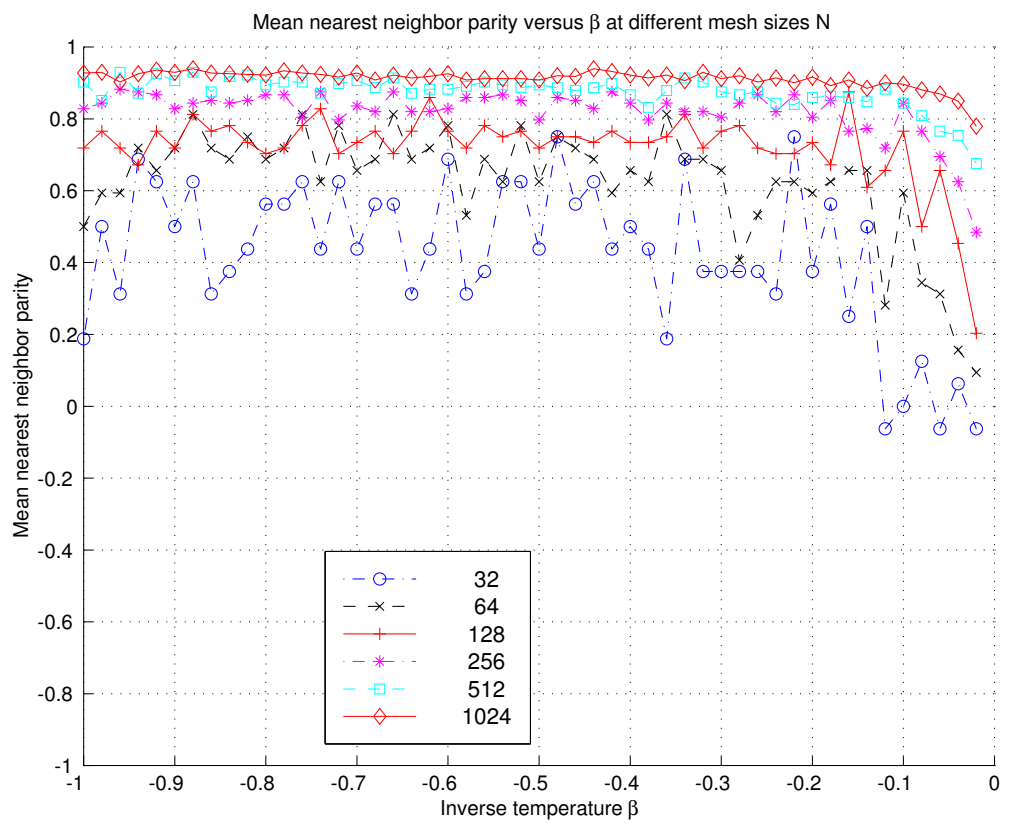

Figure 6. Mean nearest neighbor parity versus negative $\beta$ for different mesh sizes $N$.

that the system could be driven to produce a mean nearest neighbor parity much less than that.

It is expected from the analyses of Lim [7] that the negative temperature state should see solid-body rotation - the vorticity over the sphere behaving like the first spherical harmonic. This is observed for negative temperatures. An example is included in figure 8. The sphere was divided into bands representing an equal angular separation from the axis between the north and the south vorticity poles. Within each band was calculated the mean angular separation between mesh sites in that band and the north-south axis, and the mean vorticities for the mesh sites in that band. This mean vorticity is then plotted as a function from 0 to $2 \pi$, representing the angle from the north vorticity pole.

Plotted on the same axes in figure 8 is a cosine wave, the function $A \cdot \cos (\theta)$, with $A$ chosen to be the maximum absolute value of any one site and $\theta$ the angle from the north vorticity pole. The agreement between the measured vorticity and the estimated cosine wave is excellent, suggesting that this parallel state is the first spherical harmonic.

From this it appears the negative temperature settles into a solid-body state and that state is the first spherical harmonic as predicted. With some curve-fitting we can even find the harmonic which best approximates this particular solid-body rotation: the vorticity is approximately

$$
\begin{aligned}
f(\vec{x}) & =A \cdot Y_{1}^{0}(\operatorname{acos}(\vec{n} \cdot \vec{x})) \\
& =A \cdot(\vec{n} \cdot \vec{x})
\end{aligned}
$$




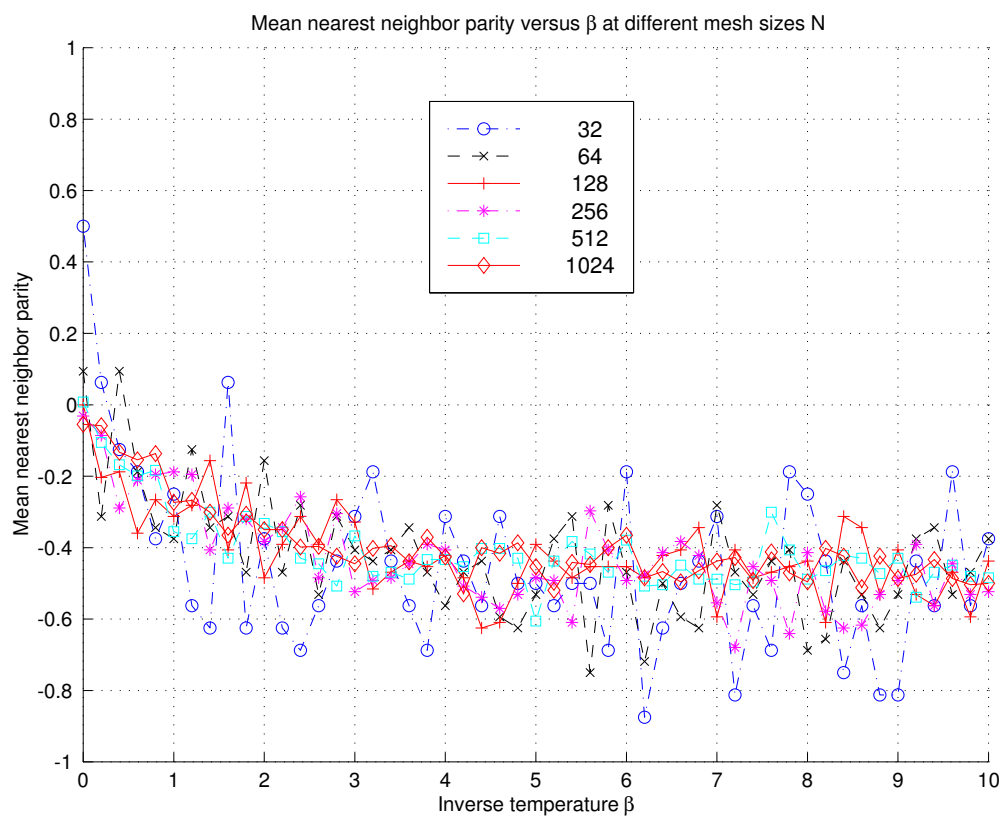

Figure 7. Mean nearest neighbor parity versus positive $\beta$ for different mesh sizes $N$.

with $\vec{n}$ the unit vector from the south vorticity pole to the north vorticity pole. The axis of rotational symmetry for the rotated spherical harmonic is parallel to the axis from the north vorticity pole to the south vorticity pole of the corresponding solidbody state. The result are two functions which agree closely. The two are plotted in figure 9.

One also may compare the energy of these solid-body states to the energy which would be expected if each mesh site had the value the first spherical harmonic would predict. These are compared for the various mesh sizes in figure 10; the figures agree well.

The good experience with fitting a spherical harmonic to the solid-body state suggests this offers a better tool to distinguish chaotic from antiparallel states: finding the spherical harmonic functions which approximate the chaotic state should find all wavelengths represented, while the antiparallel state should see the shortest wavelengths dominant [7]. This approach does not work.

We expect in the antiparallel state to see oscillations with a wavelength approximately twice the distance between nearest points. But these wavelengths are at the limit of what could be calculated even in theory. More, the distance between nearest neighboring sites varies - the result being that the 'shortest' wavelength which might be expected to have the greatest amplitude will be spread out over a range of different values. The result is that these spectral methods do not work efficiently in distinguishing the antiparallel from the chaotic states. 


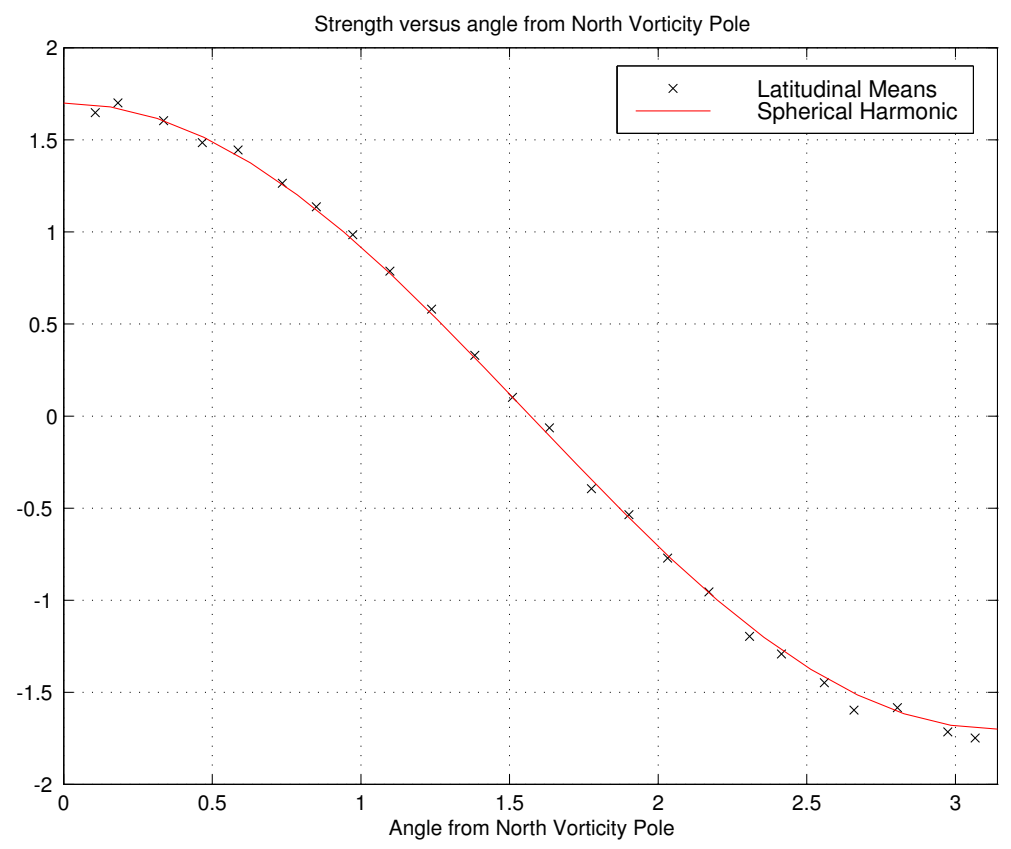

FiguRE 8. Vorticity along a great circle connecting the north and south vorticity poles. The "latitudinal means" represent the mean angular separation from the north pole for the mesh sites in a given band, and the mean site strengths for the mesh sites within that same band.

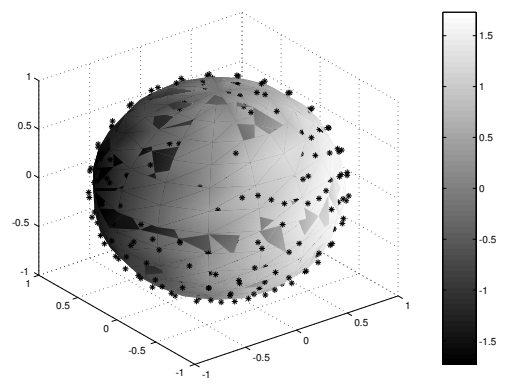

a.

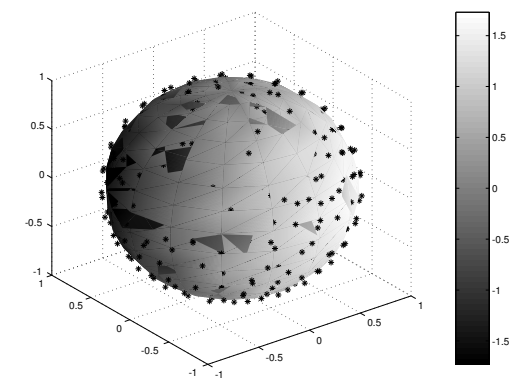

b.

FiguRe 9. A solid-body state (left) compared to a spherical harmonic (right), on the same mesh and both with 'axes' from the south to the north vorticity poles parallel.

\section{Conclusions}

Monte Carlo simulation of the spherical model finds evidence for the existence of three states for the system, which may roughly be classified by the likelihood a site has as its neighbors sites of the same or of the opposite sign. These present 


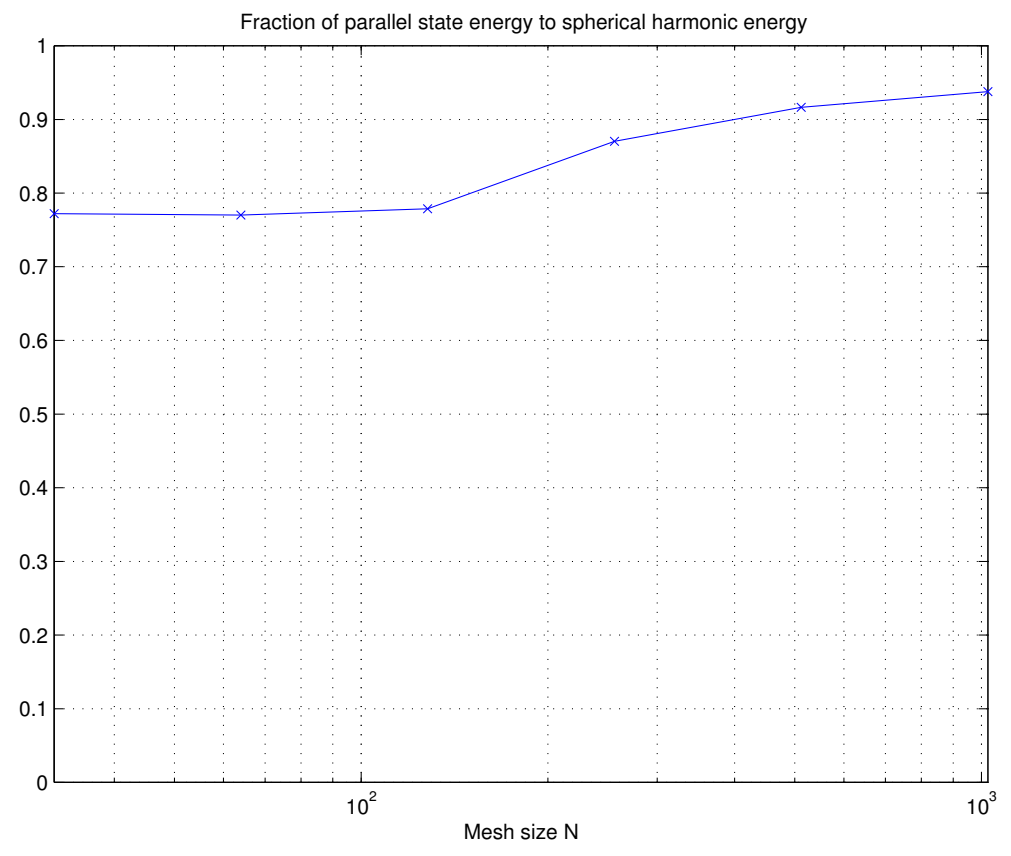

FiguRE 10. Energy of the parallel state as a fraction of the spherical harmonic's energy on the same mesh.

two organized states, one with most sites parallel to their neighbors and one with most sites antiparallel to their neighbors, and one disorganized state.

It is not difficult to distinguish the parallel state from the two others. It has a large positive value for the mean nearest neighbor parity. It has the distance between the greatest positive and the greatest negative sites close to the diameter of the sphere. Its site values at any time after it has settled into the state are more uniformly distributed than the other states are. It can be approximated very well by a simple spherical harmonic. Finally, its energy is quite high.

It is distinguishing between the chaotic and the antiparallel states that is more difficult. Study through spherical harmonics is unproductive. The distance between the north and south vorticity poles for a single state cannot be relied upon, although its evolution over a number of sweeps is unmistakable. The mean nearest neighbor parity offers some guidance but it can be mislead by the number of states with near-zero values which are able to drive its value towards zero. The magnitude of its energy for any one state is not conclusive either.

An historical record of site values over the course of a simulation would distinguish between a chaotic and an antiparallel state in the cases when the mean nearest neighbor parity is ambiguous: the chaotic system sees wider fluctuations in the energy and in any one site's values, while the antiparallel one sees a settled energy and a tight bell curve of any one site's values. That this suggests a single collection of site values could not have its state measured underscores that the mean nearest neighbor parity can be used to distinguish between different states, but does not by itself describe the behavior of the system. There is no cutoff that a system 
with mean nearest neighbor parity less than some chosen border is antiparallel; but the progression of mean nearest neighbor parities as another parameter is changed gives some insight into how the behavior of the system depends on that parameter.

Nevertheless the trend is always for the mean nearest neighbor parity to be a large positive number for negative $\beta$, falling towards zero as the inverse temperature approaches zero, and then continue falling towards -1 as for positive $\beta$. In contrast to the analysis in [7], this is supportive of a single phase transition occurring at $\beta=0$, the transition between negative and positive temperatures and the transition between the parallel and antiparallel states. A revised study in [9] confirms this conclusion of a single phase transition.

Though the values of the mean nearest neighbor parity fluctuate as the number of Monte Carlo sweeps increases, the size of those fluctuations remains small after the first few thousand sweeps - after a short settling period, essentially - and the size of those fluctuations decreases as the number of points $N$ increases.

\section{ACKnowledgements}

This research was partially supported by grant R-151-000-015-112 at the National University of Singapore. We would like to thank the faculty and staff of Computational Science at the NUS and the Dean of Science of the NUS for their hospitality and support of this work.

\section{REFERENCES}

[1] T.H. Berlin and M. Kac. The spherical model of a ferromagnet. Phys. Rev., 86 (1952) 821-835.

[2] E. Caglioti, P. L. Lions, C. Marchioro, M. Pulvirenti, A special class of stationary flows for two-dimensional Euler equations: a statistical mechanics description. Comm. Math. Phys. 143 (1992) 501-525.

[3] A.J. Chorin, J.E. Marsden. A Mathematical Introduction to Fluid Mechanics. Springer-Verlag New York City, 1979.

[4] J. M. Hammersley and D. C. Handscomb, Monte Carlo Methods. Methuen \& Co, London; John Wiley \& Sons, New York City, 1964.

[5] R. Kidambi and P. Newton, Motion of three point vortices on a sphere. Physica D 116 (1998) 143-175.

[6] Chjan C. Lim, A long range Spherical model and Exact non-Gaussian Solutions of a EnergyEnstrophy Theory for 2-D Turbulence. Phys. Fluids 13 (2001) 1961-1973.

[7] Chjan C. Lim, Exact solutions of an energy-enstrophy theory for the barotropic vorticity equation on a rotating sphere. Physica A 290 (2001) pages 131-158.

[8] Chjan C. Lim, Joseph Nebus, Syed M. Assad. Monte-Carlo and Polyhedron-based Simulations I: Extremal States of the Logarithmic N-Body Problem on a Sphere. Discrete and Continuous Dynamical Systems B 3 (2003) 313-342.

[9] Chjan C. Lim, Phase Transitions and Coherent Structures in an Energy-Enstrophy Theory for Axisymmetric Flows. Phys. Fluids, 15 (2003) 478-487.

[10] L. Onsager, Statistical Hydrodynamics, Nuovo Cimento Suppl. 6 (1949) 279-289.

[11] H. E. Stanley, Spherical model as the limit of infinite spin dimensionality. Phys. Rev. 176 (1968) 718-722.

E-mail address: Chjan C. Lim, limc@rpi.edu.

Department of Mathematical Sciences, Rensselaer Polytechnic Institute

Department of Computational Science, National University of Singapore 